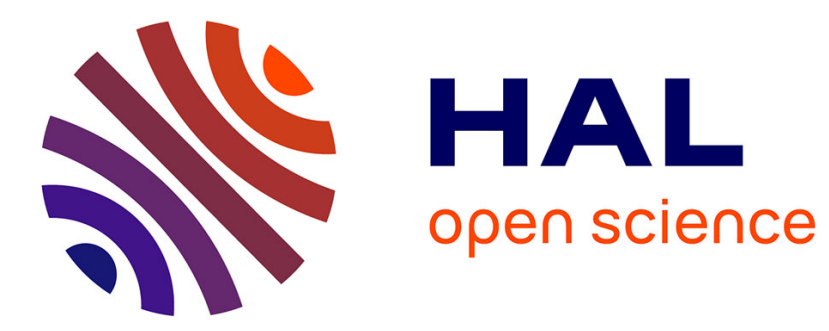

\title{
Ionospheric storms on Mars: Impact of the corotating interaction region
}

\author{
E. Dubinin, M. Fraenz, J. Woch, F. Duru, D. Gurnett, Ronan Modolo, S.
}

Barabash, R. Lundin

\section{- To cite this version:}

E. Dubinin, M. Fraenz, J. Woch, F. Duru, D. Gurnett, et al.. Ionospheric storms on Mars: Impact of the corotating interaction region . Geophysical Research Letters, 2009, 36 (1), pp.L01105. 10.1029/2008GL036559 . hal-00364354

\section{HAL Id: hal-00364354 \\ https://hal.science/hal-00364354}

Submitted on 24 Feb 2016

HAL is a multi-disciplinary open access archive for the deposit and dissemination of scientific research documents, whether they are published or not. The documents may come from teaching and research institutions in France or abroad, or from public or private research centers.
L'archive ouverte pluridisciplinaire HAL, est destinée au dépôt et à la diffusion de documents scientifiques de niveau recherche, publiés ou non, émanant des établissements d'enseignement et de recherche français ou étrangers, des laboratoires publics ou privés. 


\title{
Ionospheric storms on Mars: Impact of the corotating interaction region
}

\author{
E. Dubinin, ${ }^{1}$ M. Fraenz, ${ }^{1}$ J. Woch, ${ }^{1}$ F. Duru, ${ }^{2}$ D. Gurnett, ${ }^{2}$ R. Modolo, ${ }^{3}$ S. Barabash,${ }^{4}$ and \\ R. Lundin ${ }^{4}$
}

Received 3 November 2008; revised 24 November 2008; accepted 1 December 2008; published 7 January 2009.

[1] Measurements made by the ASPERA-3 and MARSIS experiments on Mars Express have shown, for the first time, that space weather effects related to the impact of a dense and high pressure solar wind (corotating interaction region) on Mars cause strong perturbations in the martian induced magnetosphere and ionosphere. The magnetic barrier formed by pile-up of the draped interplanetary magnetic field ceases to be a shield for the incoming solar wind. Large blobs of solar wind plasma penetrate to the magnetosphere and sweep out dense plasma from the ionosphere. The topside martian ionosphere becomes very fragmented consisting of intermittent cold/low energy and energized plasmas. The scavenging effect caused by the intrusions of solar wind plasma clouds enhances significantly (by a factor of $\geq 10$ ) the losses of volatile material from Mars. Citation: Dubinin, E., M. Fraenz, J. Woch, F. Duru, D. Gurnett, R. Modolo, S. Barabash, and R. Lundin (2009), Ionospheric storms on Mars: Impact of the corotating interaction region, Geophys. Res. Lett., 36, L01105, doi:10.1029/2008GL036559.

\section{Introduction}

[2] The interaction of high and low-speed solar wind streams create regions of enhanced plasma density and magnetic field known as Corotating Interaction Regions (CIRs) [Hundhausen, 1972]. When these regions containing much higher densities and pressures collide with the magnetospheres of planets, they strongly affect the planetary environment. At Earth, they trigger the recurrent geomagnetic storms with various space weather effects, e.g. the $F 2$ layer in the ionosphere becomes fragmented and may even disappear. In contrast to Earth, whose atmosphere and ionosphere are shielded by a huge magnetic screen (intrinsic magnetosphere), the martian ionosphere lacking such a shield, is directly exposed to the incoming solar wind. As a result, the solar wind gradually eroded the martian atmosphere [Lundin et al., 1989, 2004]. However, a recent study performed on the Mars Express (MEX) spacecraft has shown that the escape rate of atmospheric volatiles $\left(\mathrm{H}_{2} \mathrm{O}, \mathrm{CO}_{2}\right)$ on Mars is rather modest $\left(\sim 4 \cdot 10^{23} \mathrm{~s}^{-1}-\right.$ $3.3 \cdot 10^{24} \mathrm{~s}^{-1}$ ) [Barabash et al., 2007; Lundin et al., 2008]. One of the reasons is that although, at present, Mars

\footnotetext{
${ }^{1}$ Max Planck Institute for Solar System Research, Katlenburg-Lindau, Germany.

${ }^{2}$ Department of Physics and Astronomy, Iowa University, Iowa City, Iowa, USA.

${ }^{3}$ CETP-IPSL, Velizy, France.

${ }^{4}$ Swedish Institute of Space Physics, Kiruna, Sweden.
}

Copyright 2009 by the American Geophysical Union. 0094-8276/09/2008GL036559 does not possess a global intrinsic magnetic field, the induced magnetosphere formed by pile-up of the interplanetary magnetic field (IMF) generally rather efficiently screens the planet [Dubinin et al., 2006]. The draping of the IMF increases the magnetic pressure on the dayside, just sufficiently to balance the solar wind dynamic pressure and terminate a solar wind at a certain distance from Mars [Dubinin et al., 2008a, 2008b], with the effect that only a minor fraction of the solar wind enters the ionosphere (note here that a certain fraction of the momentum flux is transported inward and transferred to planetary ions due to the magnetic field stresses). However, space weather effects can strongly affect the martian environment. For example, X-ray emissions from solar flares cause an enhancement in the low altitude ionosphere [Mendillo et al., 2006]. Enhancements in the ionospheric ionization can be also caused by high energetic particles accelerated by the shocks associated with CIRs [Morgan et al., 2009]. Dynamics of the upper ionospheric levels, which is mostly controlled by transport processes induced by solar wind interaction, can be also affected. Indeed, the ionosphere of another nonmagnetized planet - Venus, with a similar type of solar wind interaction, exhibits clear responses to solar wind forcing. The ionosphere appeared to contract and expand in response to a CIR passage [Taylor et al., 1985]. Nightside ionosphere disappearances under high solar wind pressure [Cravens et al., 1982] are also a manifestation of space weather effects at Venus. Recent observations on Venus Express show enhancements in ion escape related to interplanetary coronal mass ejections [Luhmann et al., 2008].

[3] Here we present the first study of the interaction of the solar wind and the upper ionosphere of Mars during the passage of a CIR using measurements by the Analyzer of Space Plasma and Energetic Atoms (ASPERA-3) and by the Mars Advanced Radar for Subsurface and Ionospheric Sounding (MARSIS) experiments on Mars Express. The observations were made at quite time of the solar wind cycle when CIRs are well-developed and are the dominant interplanetary disturbances outside of $\sim 1 \mathrm{AU}$.

\section{Observations}

[4] The MEX spacecraft is in a highly eccentric polar orbit around Mars with periapsis and apoapsis altitudes of about 275 and $10000 \mathrm{~km}$, respectively. The ASPERA-3 experiment is a set of in-situ and remote diagnostics, comprising the Ion Mass Analyzer (IMA), Electron Spectrometer (ELS) and two Energetic Neutral Atom (ENA) detectors [Barabash et al., 2006]. IMA measures composition $(m / q)$, energy $(E / q)$ and angular distribution of ions in the energy range $\approx 10 \mathrm{eV}-30 \mathrm{keV}$ with a field of view of 

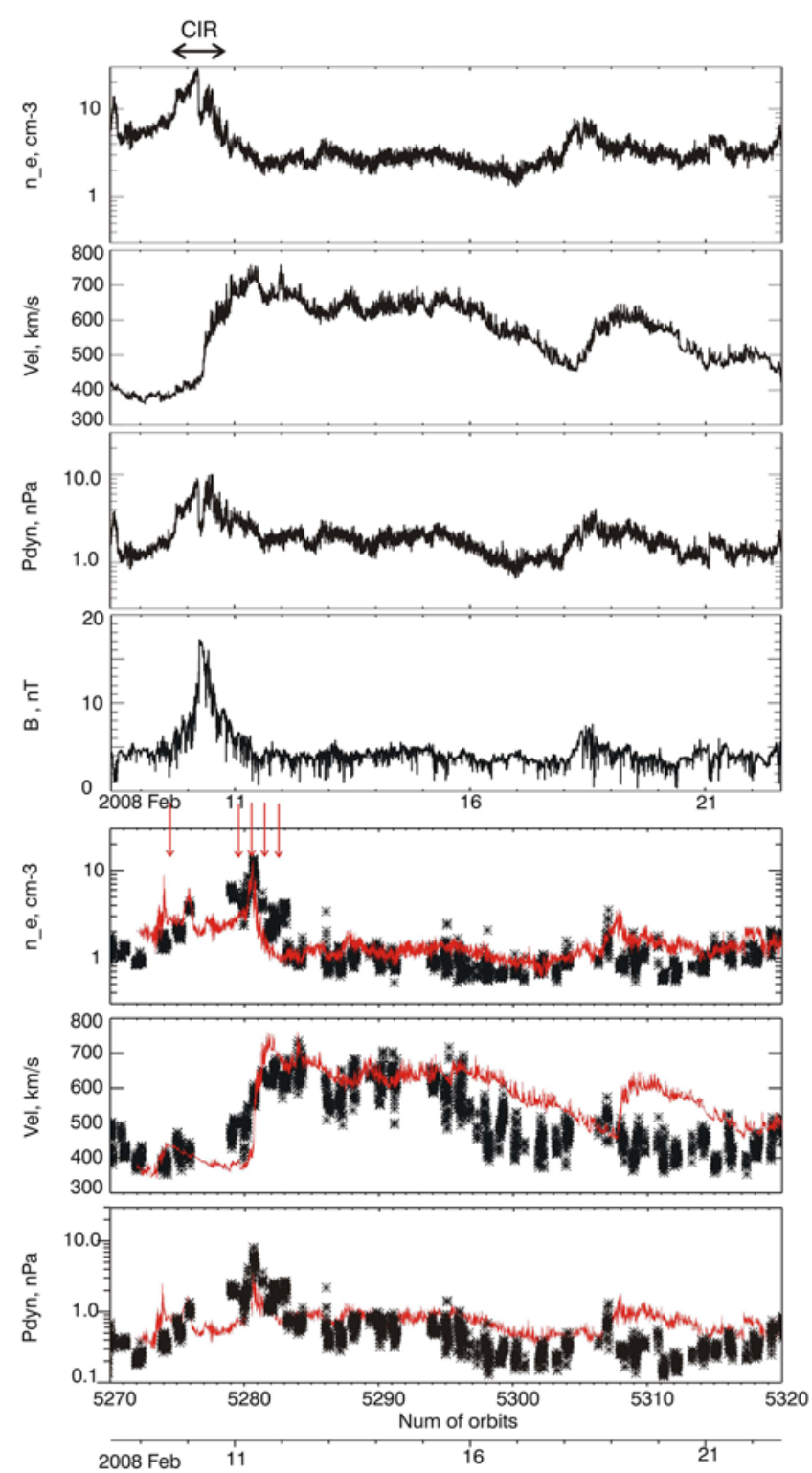

Figure 1. The top four plots show parameters of solar wind and the IMF intensity from ACE data. The bottom four plots show solar wind data near Mars from ASPERA-3. Red curves are the ACE parameters adjusted to the Mars orbit. Red arrows show the time of the MEX periapsis on the orbits discussed in the text.

$180^{\circ} \times 360^{\circ}$ achieved by electrostatic scanning in the elevation direction. The measurements of the cold/low energy component $(E \leq 50 \mathrm{eV})$ are carried out without the elevation scan with a field of view of $4^{\circ} \times 360^{\circ}$. The time resolution for the measurements of the cold and hot ion components is, respectively, $16 \mathrm{~s}$ and $192 \mathrm{~s}$. The ELS sensor measures a 2D distribution of electron fluxes in the energy range $5 \mathrm{eV}-20 \mathrm{keV}$ with a time resolution of $4 \mathrm{~s}$. MARSIS carries out not only remote sounding measurements of ionospheric height density profiles, but also, with some constraints, in-situ measurements of the local electron number density and the magnetic field strength inferred from detected plasma wave echoes [Gurnett et al., 2005; Duru et al., 2008].
[5] Figure 1 compares the solar wind parameters during February 2008 measured by the monitoring Advanced Composition Explorer (ACE) spacecraft which orbits the L1 equilibrium libration point at about 1.5 million $\mathrm{km}$ from Earth, and by ASPERA-3 in Mars orbit. During this time interval Mars was at $\sim 25^{\circ}$ with respect to the Sun-Earth line and was immersed in approximately the same expanding solar wind plasma. The CIR bounding the streams of the fast and slow solar wind was observed by both spacecraft. ACE detected the CIR on Feb. 10 when the solar wind density and the magnetic field strength increased up to $\sim 30 \mathrm{~cm}^{-3}$ and $17 \mathrm{nT}$, respectively (top four plots). The CIR reached the Mars orbit on Feb. 11. The solar wind density $\left(n_{e}\right)$, velocity $\left(V_{\alpha}\right)$ and dynamic pressure $\left(m_{p} n_{e} V_{\alpha}^{2}\right)$, where $m_{p}, n_{e}, V_{\alpha}$ are, respectively, the proton mass, the electron number density and the speed of alpha particles, are shown on the bottom three plots. The red curves present the ACE data adjusted to the Mars orbit. On Feb. 9-11, 2008 the periapsis of MEX was at a solar zenith angle (SZA) of $\sim 43^{\circ}$ near the ecliptic plane. Figure 2 shows the energy-time spectrogram of the electron fluxes measured by ELS for nominal conditions (Feb. 9) and on four subsequent MEX orbits on Feb. 11 during the CIR passage. The periapsis times of these orbits are shown by red arrows in Figure 1. ELS provides us with information about the solar wind component $(E \geq 5 \mathrm{eV})$. On orbit 5274 the magnetospheric cavity, void of solar wind plasma and marked by a drop of fluxes of the magnetosheath electrons (MB), is rather broad. The ionosphere is well recognized from the $\mathrm{CO}_{2}$ photoelectron peaks observed at $\sim 20 \mathrm{eV}$. However, most of the cold and dense ionospheric plasma is missed by ELS. On the other hand, MARSIS measures the total electron density in the regions where plasma wave echoes from the radar sounding pulses are detected (the superposed black curve). The density at the periapsis of the orbit is about $2 \cdot 10^{3} \mathrm{~cm}^{-3}$. The spike of the sheath electrons at $\sim 15: 41$ UT probably corresponds to the crossing of the plasma sheet (PS). This rather typical picture for the MEX observations is drastically changed when the dense and high pressure solar wind of the CIR impacts the magnetosphere. On orbit 5279 the magnetospheric cavity shrinks and the ionosphere becomes much weaker. The peak density is only about $\sim 2 \cdot 10^{2} \mathrm{~cm}^{-3}$.

[6] Although the magnetospheric boundaries (MB) could be still tentatively determined from the ELS observations on orbit 5280 when the peak of the CIR crossed the Mars orbit, the observations of multiple clouds of solar wind plasma within the cavity show that the 'permeability' of the induced magnetosphere significantly increases. The ionosphere is also very strongly affected. Large pieces of ionospheric plasma are swept out by the intruding solar wind clouds resulting in a drastic erosion of the ionosphere. On the next orbit (5281) the magnetosphere somewhat 'recovers' although it still contains a lot of smaller scale intrusions. Later (orbit 5282), the martian magnetosphere has almost recovered to the nominal state. It is void of solar wind plasma except for a spike of magnetosheath like electrons ( $\sim 22: 18$ UT) which probably corresponds to the PS crossing. A drop in the density in the PS is probably caused by the enhanced horizontal transport of ionospheric plasma due to the magnetic field tension of the draped IMF. The measured flux of escaping oxygen ions in such a channel is about $5 \cdot 10^{7} \mathrm{~cm}^{-2} \mathrm{~s}^{-1}\left(n_{e} \sim 25 \mathrm{~cm}^{-3}, V_{O+} \sim 20 \mathrm{~km} / \mathrm{s}\right)$. 

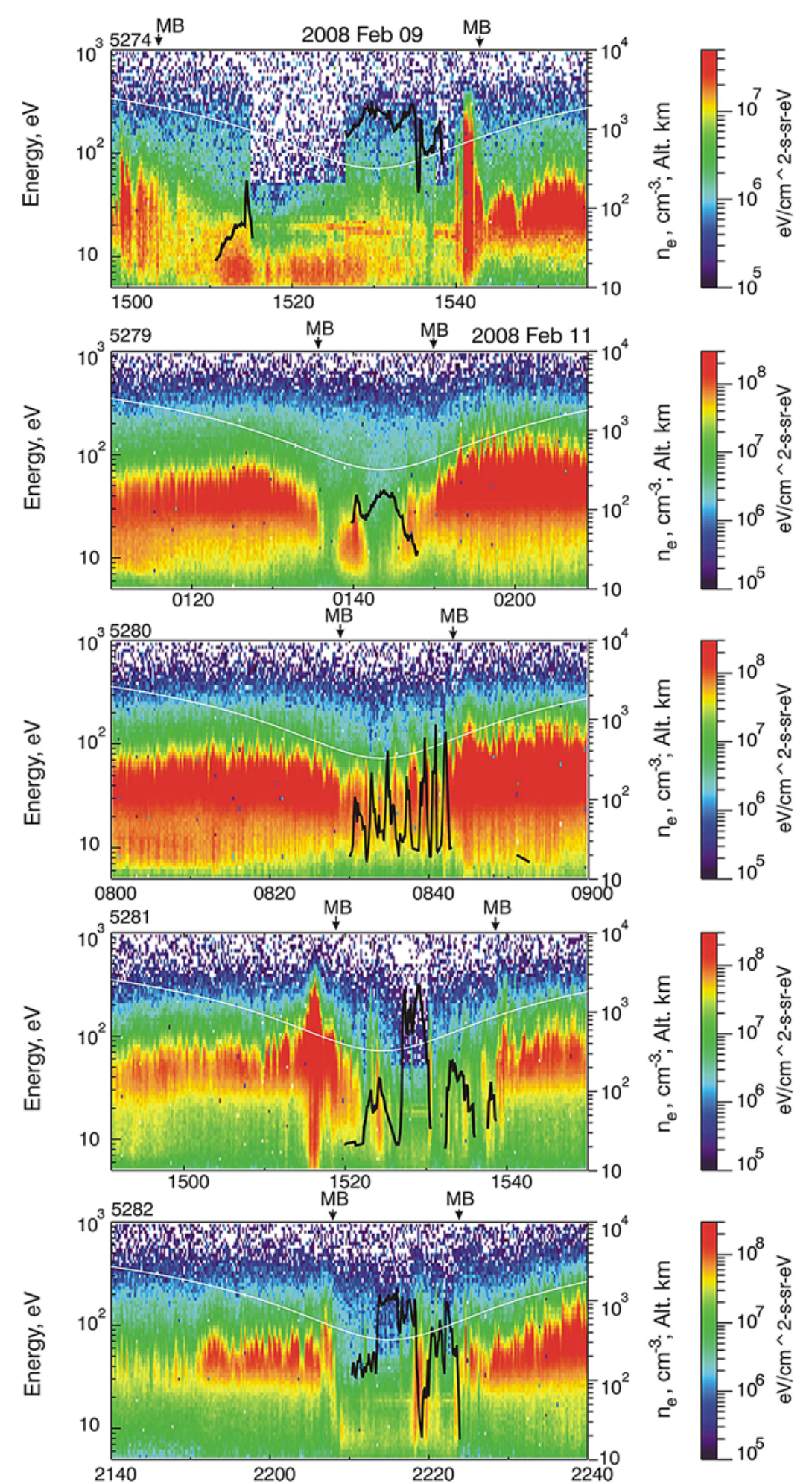

Figure 2. Spectrograms of electron fluxes which show the dynamics of the martian magnetosphere during the passage of the CIR. The superposed black curves are the electron number densities inferred from the MARSIS observations. The white curves show the altitude of MEX. 

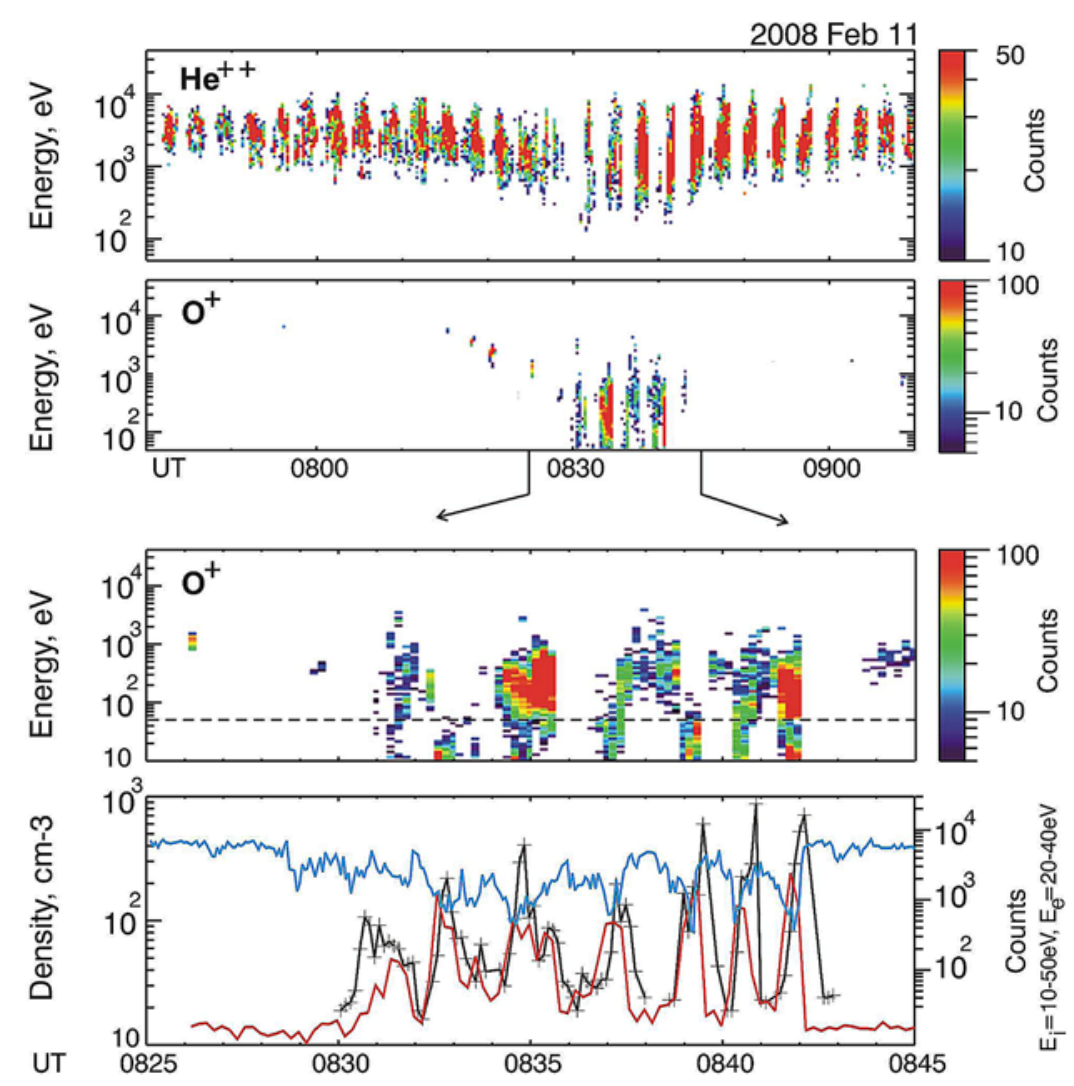

Figure 3. Spectrograms of ion $\left(H e^{++}, \mathrm{O}^{+}\right)$fluxes on orbit 5280. The bottom plot depicts the total electron density (symbols) inferred from the MARSIS observations. Red and blue curves show the fluxes of low energy $(10-50 \mathrm{eV})$ oxygen ions and solar wind electrons $(20-40 \mathrm{eV})$, respectively.

An enhanced permeability of the induced magnetosphere and fragmentation of the ionosphere arising from a CIR impact is also clearly seen from the ion observations. Figure 3 presents the energy-time spectrograms of ion fluxes. The top two plots show the spectrograms of solar wind $\left(\mathrm{He}^{++}\right)$and planetary $\left(\mathrm{O}^{+}\right)$ions which were measured in the electrostatic 'steering' mode. The duration of one scan is about 3 min which results in spotted features of alpha particles and energized oxygen ions on the spectrograms. In contrast, spots in the low-energy component are caused by a fragmentation of the ionospheric plasma (IMA operates without scanning in the low energy range). The third plot from the top depicts in more detail spectra of $O^{+}$ions measured in scanning $\left(E_{i} \geq 50 \mathrm{eV}\right)$ and nonscanning $\left(E_{i}<\right.$ $50 \mathrm{eV}$ ) modes. Comparison with the MARSIS data confirms a strong ionospheric structuring. The black and red curves on the bottom plot show the total electron number density inferred from the MARSIS measurements and the total counts of oxygen ions in the energy range of $10-50 \mathrm{eV}$, respectively. Both curves correlate rather well confirming the appearance of dips in the ionospheric density at the times (or locations) when (or where) the intruded clouds of solar wind plasma, which are marked well by the blue curve showing the total counts of the electron fluxes $\left(E_{e}=20-\right.$ $40 \mathrm{eV}$ ), scavenge the ionospheric plasma. The peaks (dips) in the intrusions correspond to dips (peaks) in the ionospheric number density.

[7] Momentum exchange between both plasmas leads to the energization of heavy ionospheric ions. Local ionospheric plasma serves as a load for moving solar wind clouds [Lundin and Dubinin, 1985]. The velocity of $O^{+}$ions in the centers of the ionospheric troughs $(\sim 60 \mathrm{~km} / \mathrm{s})$ is still less than the speed of the intruded plasma that corresponds to the regime of 'loaded pickup' [Lundin and Dubinin, 1992]. A classical pick-up population is also observed (08:05-08:30 UT) in the boundary layer and magnetosheath.

\section{Discussion}

[8] The detection of CIR effects in the topside ionosphere may have important consequences. Generally, a fragmentary pattern of the ionospheric plasma does not necessarily imply a net depletion. Intrusions of solar wind plasma can also result in a redistribution of plasma in the ionosphere. However, the ion measurements show that the ionospheric ions within these regions gain the energy well above the escape energy. We can roughly estimate the ionospheric losses. Figure 4 shows a sketch of the transverse $\left(Y Z_{M S O}\right)$ cross-section of the martian ionosphere at the dayside during 'quiet' solar wind conditions (a) and during the passage of a CIR (c). The cross-flow component of the IMF determines the draping configuration of the induced magnetosphere and the orientation of the plasma sheet. Shaded areas in Figure 4a correspond to the sites with the largest curvature of the draped field lines. Under the action of the magnetic field stresses the ionospheric plasma in these areas is scavenged with formation of two main erosion channels in the ionosphere which gradually develop into the 
a)

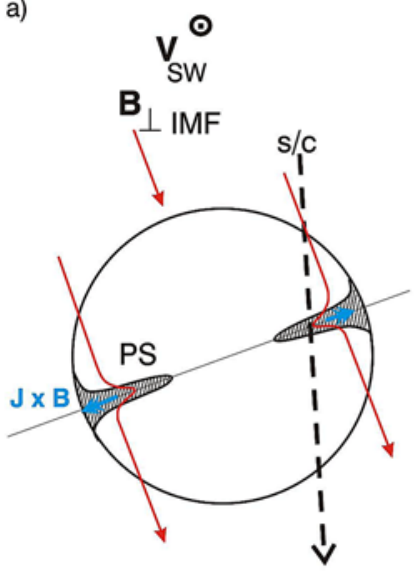

b)

$$
\mathrm{n}_{\mathrm{e}}, \mathrm{cm}^{-3}
$$

$2.510^{3}$

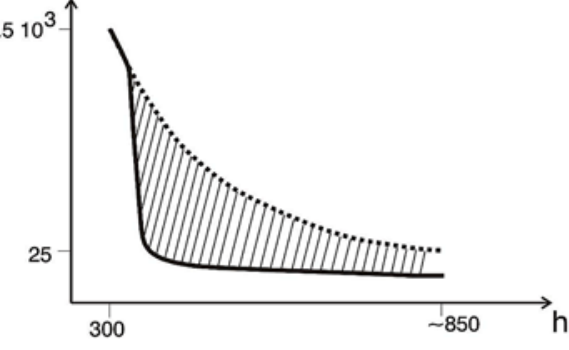

c)

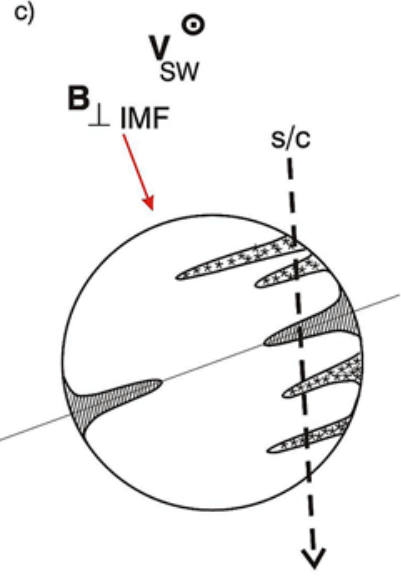

d)

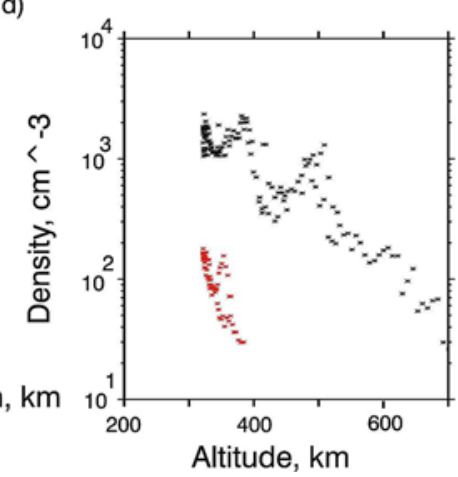

Figure 4. (a) Sketch of the martian ionosphere on the dayside in a cross-section perpendicular to the solar wind flow. Shaded areas show the depletion regions caused by scavenging of the ionospheric plasma. (b) Sketch of the height profile of the electron number density in the erosion channel. (c) Sketch of the ionosphere at the CIR impact with multiple erosion channels. (d) Altitude profiles of the electron number densities during the CIR passage.

PS on the nightside. The sketch in Figure $4 \mathrm{~b}$ shows the height profile of the electron number density in the top-side ionosphere (the dotted curve) assuming that at altitudes $\geq 300 \mathrm{~km}, n_{e} \approx n_{o} e^{-(h-h o) / H}$, where $n_{o}=2.5 \cdot 10^{3} \mathrm{~cm}^{-3}$, $\bar{h}_{o}=300 \mathrm{~km}, H=120 \mathrm{~km}$ [Duru et al., 2008]. The solid curve depicts the height profile of the density in the erosion channel. One can easily evaluate the depleted column density $P$ which corresponds to the shaded area in Figure $4 \mathrm{~b}$. With the above numbers, $P \sim 1.5 \cdot 10^{10} \mathrm{~cm}^{-2}$. Taking the width $d$ of the erosion channel along the MEX orbit as $\sim 400 \mathrm{~km}$ (orbit 5282) and the length $L$ of this erosion strip in the horizontal direction from $\mathrm{SZA}=0^{\circ}$ to $\mathrm{SZA}=90^{\circ}$ as $\sim \pi / 2 \cdot R$, where $R \sim 4000 \mathrm{~km}$, yields the total number of scavenged ions $N \sim 4 \cdot 10^{26}$. Such an amount of particles can be lost in $\sim 5 \min \left(t \sim L / V_{\text {esc }}\right.$, where $\left.V_{\text {esc }} \sim 20 \mathrm{~km} / \mathrm{s}\right)$ which corresponds to a loss rate of about $1.3 \cdot 10^{24} \mathrm{~s}^{-1}$.

[9] During the space weather event (passage of the CIR) the number of erosion channels increases (Figure 4c). The escape flux also increases and exceeds $10^{8} \mathrm{~cm}^{-2} \mathrm{~s}^{-1}$. Thus, the number of scavenged ions and the loss rate can easily reach $2 \cdot 10^{27}$ and $2 \cdot 10^{25} \mathrm{~s}^{-1}$, respectively $\left(V_{\text {esc }}\right.$ increases up to $\sim 60 \mathrm{~km} / \mathrm{s}$ ). This is a conservative estimate since the ionospheric plasma outside of intruded regions can be also driven into a global motion. Such a global erosion is indicated by a drop of the peak values of the density measured by MARSIS. Figure 4d shows for comparison the altitude profiles of $n_{e}$ on orbit 5279 (red symbols) and orbits 5274, 5281, 5282 (black symbols). The data from the latter orbits were combined and only maximum values were taken to exclude effects of local depletions. A further analysis of a total ionospheric content based on the radar sounding measurements is necessary to test more accurately ionospheric losses during space weather events.

[10] In summary, space weather events related to the passage of a dense and high pressure solar wind cause significant perturbations - ionospheric storms in the martian space environment. Schielding effects of the induced magnetic barrier are reduced and clouds of solar wind plasma penetrate to low altitudes (at least, down to the altitudes of $270-300 \mathrm{~km}$ probed by MEX). These clouds scavenge the ionospheric plasma which becomes more rarefied and very fragmentary. The perturbations driven by solar wind at the upper ionospheric levels can propagate to lower altitudes causing the appearance of transient layers observed by MARSIS [Kopf et al., 2008].

[11] Acknowledgments. Authors (E.D., M.F., and J.W.) wish to acknowledge support from DLR for supporting this work by grant FKZ $50 \mathrm{QM} 0801$. The research at the University of Iowa was funded by contract 1224107 with the Jet Propulsion Laboratory.

\section{References}

Barabash, S., et al. (2006), The analyzer of space plasma and energetic atoms (ASPERA-3) for the Mars Express mission, Space Sci. Rev., $126,113$.

Barabash, S., et al. (2007), Martian atmospheric erosion rates, Science, 315 , 501, doi:10.1126/science.1134358. 
Cravens, T. E., et al. (1982), Disappearing ionospheres on the nightside of Venus, Icarus, 51, 271.

Dubinin, E., et al. (2006), Plasma morphology at Mars, ASPERA-3 observations, Space Sci. Rev., 126, 209.

Dubinin, E., et al. (2008a), Structure and dynamics of the solar wind/ionosphere interface on Mars: MEX-ASPERA-3 and MEX-MARSIS observations, Geophys. Res. Lett., 35, L11103, doi:10.1029/2008GL033730.

Dubinin, E., et al. (2008b), Plasma environment of Mars as observed by simultaneous MEX-ASPERA-3 and MEX-MARSIS observations, J. Geophys. Res., 113, A10217, doi:10.1029/2008JA013355.

Duru, F., D. A. Gurnett, D. D. Morgan, R. Modolo, A. F. Nagy, and D. Najib (2008), Electron densities in the upper ionosphere of Mars from the excitation of electron plasma oscillations, J. Geophys. Res., 113, A07302, doi:10.1029/2008JA013073.

Gurnett, D., et al. (2005), Radar soundings of the ionosphere of Mars, Science, 310, 1929, doi:10.1126/science.1121868.

Hundhausen, A. J. (1972), Coronal Expansion and Solar Wind, Phys. Chem. Space, vol. 5, Springer, New York.

Kopf, A. J., D. A. Gurnett, D. D. Morgan, and D. L. Kirchner (2008), Transient layers in the topside ionosphere of Mars, Geophys. Res. Lett., 35, L17102, doi:10.1029/2008GL034948.

Luhmann, J. G., A. Fedorov, S. Barabash, E. Carlsson, Y. Futaana, T. L. Zhang, C. T. Russell, J. G. Lyon, S. A. Ledvina, and D. A. Brain (2008), Venus Express observations of atmospheric oxygen escape during the passage of several coronal mass ejections, J. Geophys. Res., 113, E00B04, doi:10.1029/2008JE003092.

Lundin, R., and E. Dubinin (1985), Solar wind energy transfer regions inside the dayside magnetopause. 2. Accelerated heavy ions as traces for MHD processes in the dayside boundary layer, Planet. Space Sci., 33,891 .
Lundin, R., and E. Dubinin (1992), Phobos-2 results on the ionospheric plasma escape from Mars, Adv. Space Res., 12(9), 255.

Lundin, R., et al. (1989), First results of the ionospheric plasma escape from Mars, Nature, 341, 609.

Lundin, R., et al. (2004), Solar wind-induced atmospheric erosion at Mars: First results from ASPERA-3 on Mars Express, Science, 305, 1933.

Lundin, R., S. Barabash, M. Holmstrm, H. Nilsson, M. Yamauchi, M. Fraenz, and E. M. Dubinin (2008), A comet-like escape of ionospheric plasma from Mars, Geophys. Res. Lett., 35, L18203, doi:10.1029/2008GL034811.

Mendillo, M., et al. (2006), Effects of solar frares on the ionosphere of Mars, Science, 311, 1135, doi:10.1126/science.1122099.

Morgan, D., et al. (2009), Radar absorption due to corotating interaction region encounter with Mars detected by MARSIS, Icarus, in press.

Taylor, H. A., Jr., et al. (1985), Response of Earth and Venus ionospheres to corotating solar wind streams of 3 July 1979, Earth Moon Planets, 32, 275 .

S. Barabash and R. Lundin, Swedish Institute of Space Physics, SE98128 Kiruna, Sweden. (stas@irf.se; rickard@irf.se)

E. Dubinin, M. Fraenz, and J. Woch, Max Planck Institute for Solar System Research, Katlenburg-Lindau, D-37191, Germany. (dubinin@ mps.mpg.de)

F. Duru and D. A. Gurnett, Department of Physics and Astronomy, University of Iowa, Iowa City, IA 52242, USA. (firdevs-duru@uiowa.edu; donald-gurnett@uiowa.edu)

R. Modolo, CETP, IPSL, F-78140 Velizy, France. (ronan.modolo@cetp. ipsl.fr) 